Editorial

\title{
Perspectives on adapted physical activity formation in Europe
}

\author{
Yves Vanlandewijck ${ }^{1,2}$ \\ Received: 16 $6^{\text {th }}$ April 2021; Accepted: 27 $7^{\text {th }}$ April 2021; Published: 28 ${ }^{\text {th }}$ April 2021
}

The International Federation of Adapted Physical Activity (APA) defines APA as a crossdisciplinary body of practical and theoretical knowledge directed toward impairments, activity limitations, and participation restrictions in physical activity. It is a service delivery profession and an academic field of study that supports an attitude of acceptance of individual differences, advocates access to active lifestyles and sport, as well as promotes innovative and cooperative service delivery, supports, and empowerment. Adapted physical activity includes, but is not limited to, physical education, sport, recreation, dance, creative arts, nutrition, medicine, and rehabilitation (International Federation of Adapted Physical Activity, 2021). From this definition, we can deduce that the expert in the domain of APA is a millipede, trained in multidisciplinary ways and capable of applying knowledge and skills to individuals with chronic disease and disabilities in therapeutic, education, leisure time physical activity and sport contexts.

Late Dr. Trevor Williams, professor at Loughborough University, understood that one single university could not cover this complex domain of expertise. Therefore, in 1989, during the $7^{\text {th }}$ International Symposium of APA in Berlin, he launched the European Master's Degree in APA, combining competencies of eight European universities. The consortium selected KU Leuven in Belgium as the host of the 60 credits European program. From 1991 to 2004, the program was very successful, attracting more than 25 European students per year. In 2005, a new consortium of universities successfully applied for an Erasmus Mundus grant. During the next decade from 2005 to 2014, KU Leuven hosted the Erasmus Mundus Masters in APA, opening the program to students worldwide. It was halfway through this Erasmus Mundus period that the program transformed to a 120 credits master's program. From 2015 until today, the program runs as an International Master's Program in APA, structured around the three major professional contexts in APA as described in the European Standards in APA, i.e. Adapted Physical Education, Rehabilitation, and Sport (Kudlacek, Morgulec-Adamowicz \& Verellen, 2010).

More than 400 APA-graduates over the past 30 years, predominantly engaging in professional and academic leading positions worldwide, are testimony of the success of the program. In the last three decades, the domain of APA became more popular amongst scholars, with an exponential growth in, for example, scientific peer-reviewed biomedical publications (Pubmed databank). From 1991 to 2000, only eight biomedical papers referred to "Adapted Physical Activity"; in the following decades, this number increased to 33 (2001 to 2010) and 307 (2011 to 2020). However, such criteria, to some extent indicating the growth of the domain of APA, should not make us blind for persistent issues within the domain that did not change too much compared to the situation in 1989.

In many European countries, we still struggle to transit individuals with acquired and congenital impairments from a rehabilitation or education context to leisure time physical activity and consequently to a better quality of life. Physical activity programs for those with complex health disorders such as stroke or cerebral palsy are lacking or have minimal scientific substantiation (Sammut, Fini, Haracz, Nilsson, English \& Janssen, 2020; 
Verschuren, Peterson, Balemans \& Hurvitz, 2016). A recent survey in the Flemish part of Belgium identified $37 \%$ of individuals with neuromusculoskeletal impairment as physically active, i.e. engaged in moderate physical activity at least once a week (Scheerder et al., 2018). Not only the $37 \%$ is problematic, compared to the numbers in able-bodied populations, but also the volume of physical activity is far below the criteria of healthy living (American College of Sports Medicine et al., 2017; World Health Organization, 2020). The cause of decreased active lifetime and increased sedentary behavior is individually different and demands a personalized approach starting in school or rehabilitation. Williams, Smith \& Papathomas (2014) provide an excellent framework of concepts and themes explaining the complexity of barriers and facilitators to leisure time physical activity (LTPA) in individuals with spinal cord injury. This brings us to the second issue on the table: who should be the artist to bridge the rehabilitation and education context to LTPA?

I call the instructors, guiding the transition process to LTPA, artists because of the ingenious creativity that they combine with the multidisciplinary insight in the person interacting with a dynamic environment. The profile of the artist is unique, merging competencies from physiotherapy, occupational therapy, physical education, sport sciences, training, coaching, and management, guiding individuals with chronic diseases and disabilities to LTPA (Kudlacek et al., 2010). In several European countries, both experts in rehabilitation sciences and movement sciences claim the role of movement consultants, alleging to know both sides of the bridge between rehabilitation and LTPA, between education and LTPA. However, physiotherapy formation concentrates more on the acute and subacute phase in rehabilitation, whereas physical education and sport sciences formation accentuates movement as a preventive tool in typically developing children and youngsters. Consequently, professionals miss the knowledge and skills to orient and conduct individuals with chronic diseases and disabilities to LTPA and, therefore, the transition becomes the participant's own responsibility.

In the past 30 years, the International Master program in APA accepted students holding a bachelor's degree in physiotherapy, physical education, sport sciences or an equivalent qualification. All students needed to step out of their comfort zone to acquire the knowledge and skills specific to the domain of APA. Graduating from the program, students with a physical education and sport science background faced legal obstacles entering the clinical or therapeutic environment, and students with a physiotherapy background were restricted in the pedagogical professional field. It is clear that the domain of APA would benefit from a legal framework stipulating the qualifications of the APA-expert in therapeutic, pedagogical and para-sports environments.

In September 2022, the last cohort of students will graduate from the International Master Program in APA coordinated by KU Leuven. For administrative reasons, KU Leuven decided not to continue their role as coordinator of the program. Consequently, APAformation in Europe will reshape in the upcoming months, moving the international program to a new host and adapting the format of the program to the professional and academic needs of the field. Safeguarding an efficient and effective transition from therapy to healthy lifestyle is just one of the many aspects that will make this journey challenging and exciting.

\footnotetext{
Author affiliations:

1 Department of Rehabilitation Sciences, Faculty of Kinesiology and Rehabilitation Sciences, KU Leuven, Belgium; yves.vanlandewijck@kuleuven.be

2 Department of Physiology, Nutrition and Biomechanics, Swedish School of Sport and Health Sciences (GIH), Stockholm, Sweden

* Correspondence: yves.vanlandewijck@kuleuven.be
} 


\section{References}

American College of Sports Medicine, Riebe, D., Ehrman, J. K., Liguori, G., \& Magal, M. (2018). ACSM's guidelines for exercise testing and prescription (10th edition). Philadelphia: Wolters Kluwer.

International Federation of Adapted Physical Activity. (2021). https://ifapa.net/definition/

Kudláček, M., Morgulec-Adamowicz, N., \& Verellen, J. (2010). European Standards in Adapted Physical Activity. Olomouc: Palacký University https://eufapa.eu/eupapa/eusapa/eusapa-pub/

Sammut M., Fini N., Haracz K., Nilsson M., English C., \& Janssen H. (2020). Increasing time spent engaging in moderate-to-vigorous physical activity by community-dwelling adults following a transient ischemic attack or non-disabling stroke: a systematic review. Disability and Rehabilitation, 1, 1-16. https://10.1080/09638288.2020.1768599

Scheerder, J., Vanlandewijck, Y., Biesen, D. van, Cans, E., Lenaerts, L., Meganck, A.S., \& Cornelissen, J. (2018). Research into the active sports participation of persons with a disability in Flanders and the Brussels-Capital Region: a baseline measurement. Leuven: KU Leuven.

Verschuren O., Peterson M. D., Balemans A. C. J., \& Hurvitz E. A. (2016). Exercise and physical activity recommendations for people with cerebral palsy. Developmental Medicine \& Child Neurology, 58(8), 798-808. https://doi.org/10.1111/dmcn.13053

Williams, T. L., Smith, B., \& Papathomas, A. (2014). The barriers, benefits and facilitators of leisure time physical activity among people with spinal cord injury: a meta-synthesis of qualitative findings. Health Psychology Review, 8(4), 404-425. https://doi.org/10.1080/17437199.2014.898406

World Health Organization. (2020). Guidelines of physical activity and sedentary behaviour. https://www.who.int/publications/i/item/9789240015128

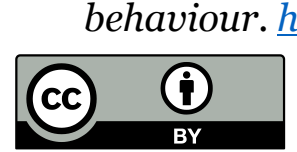

(C) 2021 by the authors. Submitted for possible open access publication under the terms and conditions of the Creative Commons Attribution (CC BY) license (http://creativecommons.org/licenses/by/4.o/). 